\title{
RESPONSE TO DR FRAZIER'S COMMENTS
}

\author{
(Research Note)
}

HAROLD ZIRIN

Big Bear Solar Observatory, California Institute of Technology, Carnegie Institution of Washington

Frazier (1972) criticized the conclusions of Veeder and Zirin (1970) and of Foukal (1971) on the relation of chromospheric morphology to magnetic fields. No sooner was this answered by Foukal and myself (Foukal and Zirin, 1972) when a second, more strident criticism by Frazier (directly preceding in this journal) of a more recent paper by myself (Zirin, 1972) was submitted. Solar physics is not furthered by the invective he has used, and further argument only makes the matter worse; but I feel I have to answer Dr Frazier's points to clarify the status of the $\mathrm{H} \alpha: B$ relations both for him and the solar physics community. Frazier specifically accuses me of (1) hasty generalization; (2) lack of proof; (3) misquotation, and (4) confusion of terms. I plead 'not guilty' to all four.

(1) Hasty generalization: the relations between $\mathrm{H} \alpha$ and $B$ have been presented in a series of papers (Zirin, 1971; Zirin, 1972; Foukal, 1971a, b; Prata, 1971) which show the development of the relations as better data became available. So far as the results of these specific papers are concerned, it is moot to discuss whether a few cinematogram-magnetogram pairs define a relationship, because great numbers of magnetograms are now available. For example, the Leighton-Smithson videomagnetograph operates, with a flip mirror, on one of the Big Bear refractors, producing hundreds of $\mathrm{H} \alpha: B$ pairs each day. No significant deviation from the rules set forth by Zirin (1972) has appeared. However, in general, there will always be a few 'best' pairs available, which will enable us to extend the relationship further. On every $\mathrm{H} \alpha: B$ pair dozens of different features may be seen. As was pointed out by Veeder and Zirin (1970), the relationship of each $\mathrm{H} \alpha: B$ feature is repeatable and typical, so that what Frazier terms 'hasty generalization' in fact provides very good rules for studying further data. It is always a difficult matter to stop and analyze the data at hand rather than wait for something better in the future. The ongoing analysis permits one to interpret currently available data and to properly channel the obtaining and interpreting of new data. For example, it was early apparent from the slow time change of $\mathrm{H} \alpha$ features and the $\mathrm{H} \alpha: B$ relations that magnetic features changed slowly. Had this been properly recognized, the new magnetographs would not have had to be designed for rapid repetition. In fact, most of these instruments are now used with relatively long intervals between magnetograms because the fields do change only slowly.

It should be obvious to Frazier that behind each set of data we have published there was considerably more that was not included because of the overcrowding of the journals. A short trip to Pasadena or an inquiry to us could have brought this before him. 
What do we presently know from all the new $\mathrm{H} \alpha: B$ data? Just what was stated in the paper Frazier attacks (Zirin, 1972). All $\mathrm{H} \alpha$ brightenings are loci of vertical field, and the brightness appears roughly proportional to the field strength, except that emerging flux shows enhanced $\mathrm{H} \alpha$ intensity. Occasionally one finds regions of enhanced $B$ which are obscured by dark $\mathrm{H} \alpha$ structures above - these are easily spotted by dark fine structure off-band or emission in the K-line. We have hundreds of $\mathrm{H} \alpha: B$ pairs to prove these results.

Frazier has gratuitously attacked the conclusion by Veeder and Zirin (1970) that areas of $p$ field around spots do not show $\mathrm{H} \alpha$ brightening. If he will take the trouble to examine the Mt. Wilson full disk magnetograms, he will find that the low resolution $\left(17^{\prime \prime}\right)$ produces a spurious $p$ region around every $p$ spot. Our conclusions flowed directly from the Mt. Wilson data, and had we ignored it he would probably have jumped us for that. The conclusion was not, as Frazier falsely states, due to an insufficient sample, as we had many, all of which showed the same result. When the first beautiful high resolution magnetogram from the San Fernando Observatory became available, we made the 'hasty', but perfectly correct generalization that the region of vertical $p$ field was limited and modified our conclusion accordingly (Zirin, 1971). One $\mathrm{H} \alpha: B$ pair was perfectly sufficient.

(2) Lack of Proof: Frazier berates us for insufficient proof that dark fibrils always are parallel to $B$. We present proof - he calls this 'not proof'; obviously there is no possibility of agreement. There are no reliable vector magnetographs at present, so we have used the deductive arguments that dark threads and fibrils may be traced to run to opposite polarity; those which cannot be followed all the way at least point to opposite polarity, in the direction the field lines might be expected to run (Foukal, 1971a). There are no exceptions!

(3) Misquotation: Frazier is legally correct in his reference to the Weart-Zirin (1969) paper, which, to avoid 'hasty generalization', stated that the EFR's (a bright $\mathrm{H} \alpha$ plage crossed by dark arched filament system) often precede the growth of large sunspot groups. However the proper term is always - all new flux erupts this way. We have hundreds of cases. For those who like statistics, a recent study by Glackin (to be published), shows that, of 250 EFR's noted on low resolution pictures, $80 \%$ appeared as new Mt. Wilson groups a few days later. We have not done the converse study to find the EFR preceding each new Mt. Wilson region and leave that useless exercise to someone else. On $80 \mathrm{~km}$ of Big Bear films we have not found a single new group which did not appear as an EFR.

To resolve Frazier's confusion on the matter of terminology, the pattern of emergence of new flux was discovered at Caltech in 1968; the regions were called 'bright regions with loops'. We then found Bruzek's (1967) earlier work, calling the dark arches or loops 'arched filament systems'. As observations accumulated it became clear that this was the unique form in which flux emerged, and I proposed (Zirin, 1971) that these features be called what they in fact were - emerging flux regions. The term EFR therefore implies (as has been clearly pointed out in the references) those regions of bright $\mathrm{H} \alpha$ plage crossed by parallel dark fibrils which we know char- 
acterize emerging magnetic flux. They are easily distinguished from ordinary fibrils by the fact that they do not disappear in off-band pictures. As the new high-resolution data reveals new solar phenomena we will unavoidably have some confusion in new terminology.

(4) Confusion of terms: by 'vertical field' I mean those places where the plages are bright and granular; by 'horizontal field' I mean those places where organized horizontal dark fibrils show the field to be predominantly horizontal. Chromospheric oscillation is restricted in those regions. By contrast, regions of no field or weak field show no fibrils and unrestricted $\mathrm{H} \alpha$ oscillation. This has been clearly defined in our work and I am sorry Frazier is confused by it.

To make a clear test of the method I successfully predicted (Zirin, 1972) magnetic fields observed by Harvey October 17, 1970 from our $\mathrm{H} \alpha$ pictures. I can think of no better test of the method than such predictions, and I find it difficult to see how Dr Frazier can say we can't deduce the field when we have in fact done it. We stand ready to do it again if he has a magnetogram to match.

\section{Acknowledgement}

This work was supported by NASA under grant NGR 05002034 and NSF under grant Ga 24015.

\section{References}

Bruzek, A.: 1967, Solar Phys. $2,451$.

Foukal, P.: 1971a, Solar Phys. 19, 59.

Foukal, P.: 1971b, Solar Phys. 20, 298.

Foukal, P.: 1971c, unpublished BBSO preprint No. 0009, 'The Relation between Chromospheric Structure, Magnetic Field and Filaments' based on invited review at Capri Conference, 1971.

Foukal, P. and Zirin, H.: 1972, this issue, p. 148.

Frazier, E. N.: 1972, this issue, p. 142.

Prata, S.: 1971, Solar Phys. 20, 310.

Veeder, G. J. and Zirin, H.: 1970, Solar Phys. 12, 391.

Weart, S. and Zirin, H.: 1969, Publ. Astron. Soc. Pacific 81, 270.

Zirin, H.: 1971, Phil. Trans. Roy. Soc. Lond. A270, 77.

Zirin, H.: 1972, Solar Phys. 22, 34. 